\title{
N ormalización de la densidad mineral ósea en niños nacidos prematuros en Viña del Mar, Chile
}

\author{
Aída Milinarsky ${ }^{1}$, Sylvia Fischer ${ }^{1}$, Vinka G iadrosich ${ }^{1,2}$, \\ María Teresa Torres ${ }^{1,3}$, Marina Arriagada ${ }^{4}$, Roberto \\ Arinoviche $^{4}$ y D unny Casanova ${ }^{1, a}$ \\ Normalization of bone mineral \\ density in premature-born children \\ from Viña del Mar, Chile
}

Background: In a previous study we reported that healthy children born at 34 or fewer gestational weeks, with adequate weight for gestational age, had not completed their bone catch-up at mean age of 6.3 years. Aim: This is a follow up report, performed on the same population one year later to determine at which age premature - born children achieve their bone mineral density (BMD) catch-up, compared to term-born controls. Material and methods: Fifteen children mean age 7 years 3 months, born at 25 to 34 weeks of gestation, with a birth weight of 740 to $2.200 \mathrm{~g}$ were studied Radius, lumbar spine and femoral neck bone mineral density, whole body bone mineral content and body composition were assessed by DEXA. Results: Height, body mass index, peripheral BMD, axial BMD, fat and lean body mass in these children were not different from term born controls. Conclusions: Premature born children with adequate weight for gestational age, achieved peripheral and axial bone mineral density catch-up at the age of 7 to 8 years (Rev Méd Chile 2007; 135: 1546-50).

(Key words: Body mass index; Bone density; Infant, premature)

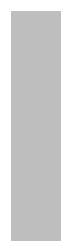

\footnotetext{
Recibido el 22 de marzo, 2007. Aceptado el 11 de julio de 2007.

Trabajo financiado por la Universidad de Valparaíso (proyecto DIPUV).

${ }^{1}$ Departamento de Pediatría. Escuela de Medicina. Universidad de Valparaíso. Valparaíso, Chile. ${ }^{2}$ Servicio de Pediatría. Hospital Carlos van Buren. Valparaíso, Chile. ${ }^{3}$ Servicio de Pediatría. Hospital Gustavo Fricke. Viña del Mar, Chile. ${ }^{4}$ Clínica de Enfermedades Reumatológicas "Clinder". Santiago, Chile.

${ }^{a}$ Estadístico. Universidad de Valparaíso. Valparaíso, Chile.
}

$\mathrm{L}$ a mineralización ósea normal durante la niñez es un proceso complejo que involucra determinantes genéticos, hormonales, nutricionales y ambientales como la actividad física y la exposi-

Correspondencia $a$ : Dra. A. Milinarsky. Escuela de Medicina. Universidad de Valparaiso. Hontaneda 2653. Valparaíso. Chile. Fax: 32-2507360. E mail: pediatria@uv.cl ción al sol, algunas enfermedades crónicas y sus tratamientos. En niños nacidos prematuros, la edad gestacional y el peso de nacimiento son importantes, ya que estos niños no completaron la acreción ósea propia de las últimas semanas intrauterinas ${ }^{1}$.

Con el avance de los cuidados intensivos neonatales, la sobrevida de los niños nacidos prematuros ha ido aumentando, por lo cual los 
pediatras deben estar atentos ante las eventuales patologías a que esto pudiese conducir en edades más tardías. Los niños nacidos prematuros tienen mayor riesgo de desarrollar déficit de la mineralización ósea, considerando que la fijación de calcio, fósforo y otros minerales ocurren especialmente en el tercer trimestre del embarazo ${ }^{1,2}$.

Se ha demostrado que la talla y el peso de estos niños se igualan a la de los niños nacidos de término en los primeros 2 años de vida. Sin embargo, lo que ocurre con la densidad mineral ósea (DMO) no está aún establecido. Algunas publicaciones describen que los niños prematuros con peso adecuado para su edad gestacional, compensan su peso, estatura y perímetro craneano durante los primeros 2 a 3 años de edad ${ }^{3-6}$. La normalización de la DMO, en cambio, según diferentes autores, se puede alcanzar en edades tan dispares como en los primeros años de vida $(7,8)$, o tan tarde como a los 8 años $^{9,10}$. En una publicación anterior ${ }^{11}$, nuestros niños nacidos prematuros a la edad promedio de 6,3 años no habían completado la normalización de la DMO evaluada por densitometría ósea periférica. El presente estudio se realizó en la misma población un año más tarde con el objeto de evaluar si habían logrado la normalización de su DMO periférica y axial. Como objetivo secundario se evaluó composición corporal.

Pacientes: Niños nacidos prematuros de la unidad de Neonatología del Hospital Carlos van Buren de Valparaíso y del Hospital Gustavo Fricke de Viña del Mar, Chile, con peso adecuado para su edad gestacional, nacidos de 34 o menos semanas de gestación, sanos, sin enfermedades congénitas de importancia, ni patología crónica respiratoria, infecciosa, digestiva, metabólica ni ósea, que constituyeron la población evaluada en el estudio previo ${ }^{11 .}$

Estos 26 niños se citaron 1 año más tarde por correo y por teléfono.

Método: A los niños que aceptaron participar en el estudio, se les hizo historia clínica para descartar patología concomitante.

La ingesta de calcio se evaluó con cuestionario sobre consumo alimentario de los 3 últimos días. La actividad física se consideró normal si los niños efectuaban actividad física escolar normal, dismi- nuida si no asistían a educación física habitual y aumentada si hacían alguna actividad física extracurricular.

En la evaluación antropométrica, se midió estatura en $\mathrm{cm}$ y se consideró el valor promedio de 3 mediciones consecutivas. Los niños se pesaron sin zapatos y con ropa liviana en balanza Seca ${ }^{\circledR}$ y el peso se expresó en Kg. Se calculó el índice de masa corporal (IMC). Todas las mediciones fueron hechas por la misma persona. Los resultados fueron expresados en puntaje $Z$ en base al grupo control.

Mediciones óseas: La DMO periférica $\left(\mathrm{g} / \mathrm{cm}^{2}\right)$ se midió con absorciometría radiológica (Osteometer DTX-100) en región radial del brazo dominante y se comparó con 40 niños sanos nacidos de término, de nuestros propios valores de referen$\mathrm{cia}^{12}$. Consideramos normal valores entre $-0,99 \mathrm{y}+$ 0,99 desviaciones estándar de los valores de referencia.

Las mediciones óseas fueron hechas por el mismo operador. El coeficiente de variación fue $0,7 \%$ in vitro, para 5 mediciones repetidas de un fantoma que entrega la fábrica. El coeficiente de variación in vivo fue de $3 \%$ para 3 mediciones repetidas en el brazo del mismo individuo en el mismo día, que se les realizó a 5 niños de diferentes edades, siguiendo el procedimiento ya descrito en publicación previa ${ }^{12,13}$.

La DMO axial fue evaluada con absorciometría radiológica (DEXA), (Lunar Prodigy) en columna lumbar, cuello femoral y cuerpo total. Se midió también contenido mineral en cuerpo completo y composición corporal y se comparó con niños de edad y talla similares. Todas las mediciones fueron hechas por el mismo operador .El coeficiente de variación fue de $0,7 \%$ in vitro, para 5 mediciones repetidas de un fantoma entregado por los fabricantes. El coeficiente de variación in vivo para mediciones repetidas en diferentes sitios del esqueleto varió entre 0,8 a $1,2 \%$.

Estadística: Se incluyó el test t de Student y MannWhitney. Se consideró el nivel alpha 0,05.

Este estudio fue aprobado por el Comité de Ética de la Escuela de Medicina, Universidad de Valparaíso, y se obtuvo consentimiento informado de los padres de los niños participantes. 
Tabla 1. D atos antropométricos y D M 0 periférica de 15 niños nacidos prematuros y sus controles

\begin{tabular}{|lccc|}
\hline & $\begin{array}{c}\text { Niños nacidos } \\
\text { prematuros } \\
\mathrm{n}=15\end{array}$ & $\begin{array}{c}\text { Niños nacidos } \\
\text { de término } \\
\mathrm{n}=40\end{array}$ & $\mathrm{p}$ \\
\hline Edad (años) & $7.3 \pm 0,6$ & $7,5 \pm 0,5$ & 0,22 \\
Talla $(\mathrm{cm})$ & $122,5 \pm 5,7$ & $124,2 \pm 5,6$ & 0,32 \\
$\mathrm{IMC}\left(\mathrm{g} / \mathrm{m}^{2}\right)$ & $16,47 \pm 2,67$ & $16,73 \pm 1,45$ & 0,56 \\
DMO $\left(\mathrm{g} / \mathrm{cm}^{2}\right)$ & $0,282 \pm 0,025$ & $0,298 \pm 0,032$ & 0,08 \\
Z DMO & $-0,05 \pm 0,02$ & 0.000 & 0,9 \\
\hline
\end{tabular}

* Desviación estándar.

\section{Resultados}

Fueron evaluados 15 niños nacidos prematuros, 7 niños y 8 niñas, promedio de edad 7 años 3 meses.

La edad gestacional fue 25 a 34 semanas, promedio $32 \pm 1,8$ con pesos de nacimiento entre $740 \mathrm{~g}$ a $2.200 \mathrm{~g}$, promedio $1.860 \pm 240$

La ingesta de calcio fue adecuada para la edad, el promedio fue $760 \pm 150 \mathrm{mg} /$ día. Todos desarrollaban actividad física normal. Las mediciones antropométricas de los 15 niños nacidos prematuros estudiados con DMO periférica mostraron un promedio de $Z$-score de talla $-0,08 \pm 0,7$, de IMC
$0,32 \pm 1,08$. El promedio de los $Z$ score de DMO fue $0,64 \pm 0,93$. Todos estos valores están en rangos de normalidad. En la Tabla 1 se muestran las mediciones antropométricas y de la densitometría ósea periférica de los niños nacidos prematuros y del grupo control. Las mediciones antropométricas, de DMO axial y composición corporal de 13 niños nacidos prematuros y sus controles se describen en la Tabla 2.

No hubo diferencias entre ambos grupos en talla, IMC, DMO de cadera, columna lumbar, CMO cuerpo completo, tejido magro ni porcentaje de grasa corporal.

Tabla 2. D atos antropométricos y D M O axial de 13 niños nacidos prematuros y sus controles

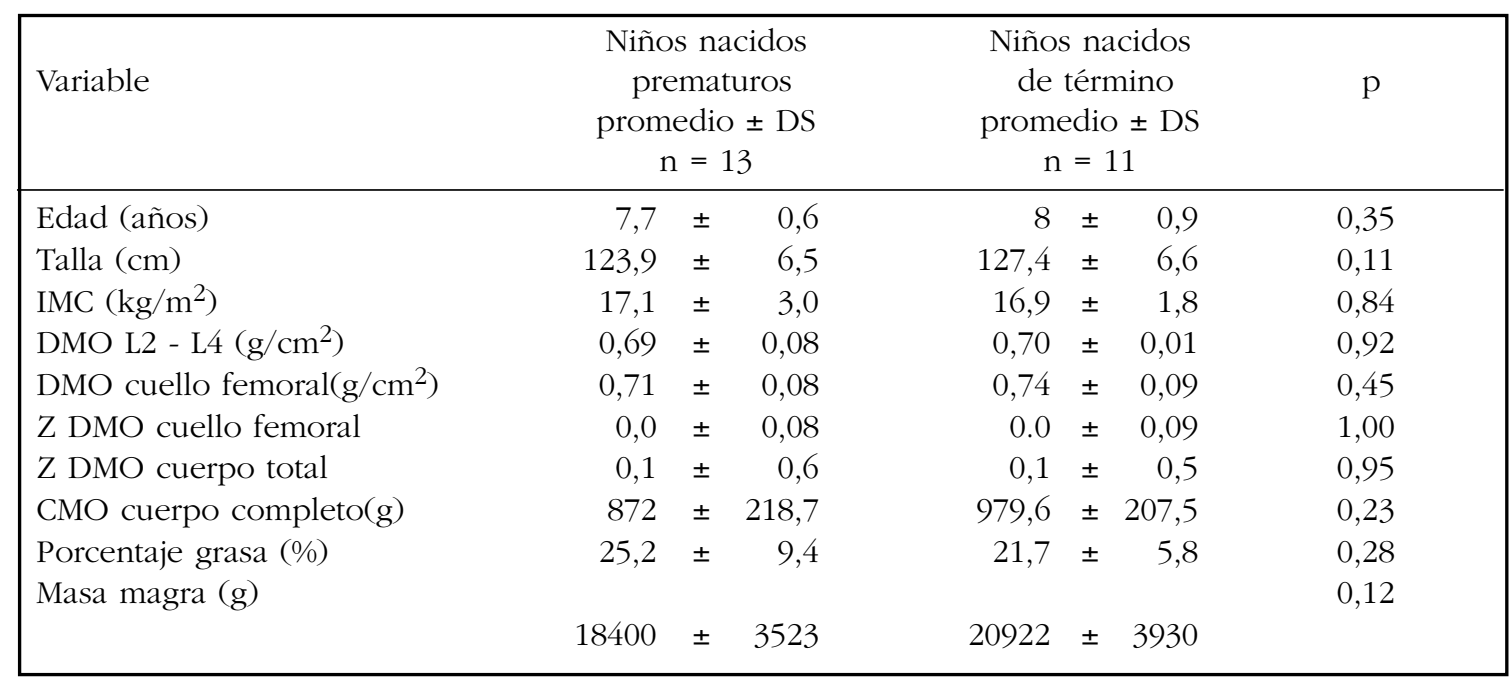




\section{DisCUSIÓN}

En una publicación anterior ${ }^{11}$, nuestros niños nacidos prematuros, con edad promedio de 6 años 3 meses, no habían incrementado su DMO a los niveles que tienen los niños nacidos de término a la misma edad, cuando fueron evaluados con densitometría periférica. En el presente reporte, que se realizó a la misma población un año después, los resultados muestran una normalización completa, tanto a nivel periférico como a nivel axial de la DMO.

El concepto densitométrico ${ }^{14}$ de normalidad en la población pediátrica es un asunto que aún no está estandarizado. Con el objeto de definir una población normal, además de tener índices antropométricos normales, es necesario tener datos de poblaciones de similar etnia, edad y sexo. La evaluación de los niños pretermino debe considerar, entre otras cosas, sus antecedentes genéticos. Nuestros niños prematuros tenían etnia similar, caucásicos, con apellidos de origen hispano en $95 \%$ de los casos, $4 \%$ de origen araucano y $1 \%$ de otros ${ }^{15}$. Se evaluó factores ambientales como ingesta de calcio, fósforo ${ }^{16}$ y vitamina $\mathrm{D}$, exposición al sol y actividad física ${ }^{17}$ y se consideraron normales. Destacamos la ingesta de calcio, que siendo normal baja, es superior a lo informado en población normal infantil chilena ${ }^{18-20}$. También es importante considerar enfermedades y medicamentos que puedan afectar el metabolismo óseo. Para este estudio seleccionamos niños sin patología crónica. .

Obviamos la influencia de las hormonas sexuales sobre el metabolismo óseo porque el grupo era prepuberal.

En el trabajo previo $^{12}$, los niños nacidos prematuros ya habían alcanzado la talla y el peso normal, lo que coincide con otros autores ${ }^{4,5}$.

\section{REFERENCIAS}

1. RYAN S. Bone Mineralization in Preterm Infants. Nutrition 1998; 14: 745-7.

2. Horsman A, Ryan SW, Congdon PJ, Truscott JG, JAMES JR. Osteopenia in extremely low-birthweight infants. Arch Dis Child 1989; 64: 485-8.

3. Congdon PJ, Horsman A, Ryan SW, Truscott JG, DURWARD H. Spontaneous resolution of bone
Comentando los resultados de la DMO, merece destacar la controversia planteada en la literatura respecto a la edad que los niños nacidos prematuros normalizan la DMO: Congdon, ${ }^{3}$ usando absorciometría de un fotón, encontró que la normalización de los huesos del antebrazo era ya completa entre las 25 y 50 semanas después de la fecha que habrían completado su gestación de término.

Ichiba, en un estudio prospectivo en niños de muy bajo peso de nacimiento, demostró que normalizan la DMO de columna lumbar a los 2 años ${ }^{7}$.

Hori $^{8}$ describió en 21 niños pretérmino, que la ingesta mineral era baja al inicio de la vida por lo que los niños se mantenían osteopénicos; sin embargo entre los 3 y 4 años todos tenían contenido mineral y DMO normales, sugiriendo que se producía una resolución espontánea de la osteopenia lumbar en los primeros años de la niñez.

Bowden describe contenido y densidad mineral ósea baja en columna lumbar, antebrazo y cuello femoral por DEXA, en niños de 8 años nacidos prematuros. Los niños tenían además en promedio 2,6 kg menos y 4,9 $\mathrm{cm}$ menos de talla?. Nuestros niños tenían la misma estatura y peso que los RN de término. La discordancia con nuestro trabajo puede reflejar la importancia del peso y la talla en el contenido y densidad mineral ósea. Este estudio de diseño longitudinal, tiene el problema de su relativo pequeño número de pacientes. Es necesario diseñar un estudio longitudinal prospectivo desde el nacimiento hasta que consigan la normalización de su DMO con adecuado número de pacientes.

En conclusión, los niños nacidos prematuros de peso adecuado para la edad gestacional, sin patología, entre los 7 y 8 años de edad, han logrado la normalización de su DMO.

mineral depletion in preterm infants. Arch Dis Child 1990; 65: 1038-42.

4. Stjernevist K, Svenningsen NW. Extremely lowbirth-weight infants less than $901 \mathrm{~g}$. Growth and development after one year of life. Acta Paediatr 1993; 82: 40-4.

5. Hack M, Merkatz IR, Mcgrath SK, Jones Pk, Fanaroff AA. Catch-up growth in very low-birthweight infants. AJDC 1984; 138: 370-5. 
6. Kitchen Wh, Doyle LW, Ford Gw, Callanan C. Very low birth weight and growth to age 8 years I: weight and height. AJDC 1992; 146: 40-5.

7. Ichiba H, Shintaku H, Fujimaru M, Hirai C, Okano Y, Funato M. Bone mineral density of the lumbar spine in very-low-birth-weight infants: longitudinal study. Eur J Pediatr 2000; 159: 215-8.

8. Hori C, Tsukahara H, Fuji Y, Kawamitsu T, Konishi $\mathrm{Y}$, Yамамото $\mathrm{K}$ eт al. Bone mineral status in preterm-born-children assessment by dual energy X-ray absorptiometry. Biol Neonate 1995; 68 : 254-8.

9. Bowden Ls, Jones Cj, Ryan Sw. Bone mineralization in preterm infant aged 8 years. Eur J Pediatr 1999; 158: 658-61.

10. Zamora SA, Belli DC, Rizzoli R, Slosman DO, Bonjour JP. Lower Femoral Neck Bone Mineral Density in Prepubertal Former Preterm Girls. Bone 2001; 29: 424-7.

11. Milinarsky A, Fischer S, Giadrosich V, Hernandez MI, Torres MT. Densidad mineral ósea en escolares nacidos prematuros. Rev Méd Chile 2003; 131: 1289-94.

12. Milinarsky A, Fischer S, Giadrosich V, Casanova D. Bone mineral density by single X-Ray absorptiometry in Chilean children and adolescent. $J$ Rheumatol 1998; 25: 2003-8.
13. Delmas PD. Bone mass measurement: How, where and why? Int J Fertil Menopausal Studies 1993; 38: 70-6.

14. Soyka LA, FAirfield W And Klibanski A. Hormonal Determinants and Disorders of Peak Bone Mass in Children. J Clin Endocrinol Metab 2000; 85: 3951-63.

15. Rocco P, Morales C, Moraga M, Miquel J, Nervi F, Llop E ET al. Composición genética de la población chilena. Distribución de polimorfismos de DNA mitocondrial en grupos originarios y en la población mixta de Santiago. Rev Méd Chile 2002; 130: 125-31.

16. NIH Consensus Development Panel on Optimal Calcium Intake. JAMA 1994; 272: 1942-8.

17. Moyer-Mileur L. Effect of physical activity on bone mineralization in premature infants. J Pedia$\operatorname{tr} 1995$; 27: 620-5.

18. Fischer S, Milinarsky A, Giadrosich V, Casanova D. Suplemento de calcio y absorciometría ósea en niñas. Rev Méd Chil 1999; 127: 23-7.

19. Leiva L, Muzzo S. Requerimientos de calcio. Rev Méd Chil 1996; 124: 29-34.

20. Leiva L, Burrows R, Lillo R, Pumarino H, Muzzo S. Mineralización ósea e ingesta de calcio en escolares chilenos. Arch Latinoam Nutr 1995; 45 : 178-82. 